\title{
Cadena de comercialización del cacao nacional en la provincia de Los Ríos, Ecuador
}

\author{
Chain of nacional cocoa marketing in Los Ríos province in Ecuador
}

Fátima Lourdes Morales Intriago ${ }^{1}$, Manuel Danilo Carrillo Zenteno ${ }^{2}$, José Ambrosio Ferreira Neto ${ }^{3}$, Mayra Mercedes Peña Galeas $^{1}$, Wilson Roberto Briones Caicedo ${ }^{1}$, Miriam Noemí Albán Moyano ${ }^{4}$

${ }^{1}$ Universidad Técnica de Babahoyo - Extensión Quevedo- Ecuador. km 3 1/2 vía Quevedo - Valencia, Teléfono: 593-052-782101.fmoralesi@utb.edu.ec,mpena@utb.edu.ec,wbriones@utb.edu.ec

${ }^{2}$ Universidad Tecnológica Equinoccial, Sede Santo Domingo - km 4 vía Santo Domingo - El Carmen. Av. Italia. mcarrillo@, ute.edu.ec Instituto Nacional de Investigaciones Agropecuarias (INIAP) Ecuador. km 5 vía Quevedo -El Empalme. manuel. carrillo@iniap.gob.ec

${ }^{3}$ Universidad Federal de Viçosa. Av. P. H. Rolfs, s/n ${ }^{\circ}$. Campus Universitario CEP: 36570-000 - Viçosa - MG. Teléfono: 55 (31) 3899-2800; correo; ambrosioufv@gmail.com

${ }^{4}$ Universidad Técnica Estatal de Quevedo km 1 via a Santo Domingo.mnalban73@hotmail.com

\section{Resumen}

$\mathrm{L}$ a producción de cacao en Ecuador es un rubro de gran importancia, genera significativas fuentes de ingresos económicos y brinda trabajo a miles de personas. El cacao Tipo Nacional conocido también como "Arriba" es producido por pequeños agricultores quienes comercializan con intermediarios, esta es una práctica habitual en el país y la provincia de Los Ríos no está al margen de esta situación. El objetivo general del trabajo fue analizar la comercialización de cacao tipo Nacional en la provincia de Los Ríos, Ecuador. De manera específica se buscó identificar, analizar y discutir los principales canales de comercialización que manejan los pequeños productores. El trabajo expresa la necesidad de intervención del Estado para que el productor comercialice directamente su producto; además, puede contribuir a empoderamiento de los productores respecto a la comercialización del cacao. Se colectaron datos cualitativos y cuantitativos. En el trabajo de campo se realizaron entrevistas a pequeños productores, revisiones bibliográficas con fundamentos teóricos e información sobre comercialización de cacao que ayudaron a comprender e interpretar los datos obtenidos. Los resultados mostraron que los pequeños productores de cacao tipo Nacional comercializan su producto por medio de intermediarios, siendo estos junto con los exportadores quienes se benefician del premio por calidad que debería ser para los pequeños productores. Se concluye que los agricultores que comercializan el cacao por medio de asociaciones reciben un valor mayor al que paga el intermediario y en estos casos, son las asociaciones quienes brindan asesoramiento e información técnica actualizada.

Palabras clave: Theobroma cacao, Intermediarios, producción, agricultores familiares
$\mathrm{T}$ The production of cocoa in Ecuador is a very important thing. It generates significant sources of economic income and provides jobs for thousands of people. Small farmers who trade with intermediaries produce the National type cocoa known also as "Arriba". This is a common practice in the country and the province of Los Ríos is not out of this situation. The general objective of the work was to analyze the commercialization of National type cacao in the province of Los Ríos, Ecuador. Specifically, we sought to identify, analyze and discuss the main marketing channels managed by small producers. The work expresses the need for intervention by the State so that the producer can commercialize the product directly. In addition, it can contribute to the empowerment of producers with respect to the commercialization of cocoa. Qualitative and quantitative data were collected. Interviews were conducted with small producers in the fieldwork. Bibliographic reviews on theoretical foundations and information on cocoa marketing helping to understand and interpret the data obtained were made. Results showed that the small producers of National type cacao market their product through intermediaries, being these together with the exporters, the ones who benefit from the quality award instead of small producers. It is concluded that farmers who commercialize cocoa through associations receive a higher value than the intermediary pays, and in these cases, the associations provide advice and updated technical information..

Keywords: Theobroma cacao, Intermediaries, production, family farmers. 


\section{Introducción}

$\mathrm{E}$ cuador es un país tradicionalmente agrícola, gran parte de su desarrollo proviene de productos como banano, cacao, café y flores (Rodríguez y Fusco, 2017). Es considerado el primer productor mundial de cacao fino y de aroma, aportando el $70 \%$, seguido de lejos por Indonesia que contribuye con un 10\% del total mundial (PROECUADOR, 2013).

Para el año 2015 Ecuador logró exportar 236,677 toneladas de cacao en grano de las cuales 165, 673 son de la calidad fino y de aroma que representa el $70 \%$ del total enviado (ANECACAO, 2015).

Este tipo de cacao es utilizado para preparar chocolates selectos y gourmets a nivel internacional, por ser calificado como el mejor del mundo (MAGAP, 2012). Debido a este reconocimiento el grano es muy apetecido y tiene gran demanda en los mercados internacionales. Es un producto que asegura una demanda significativa ya que se destaca no solo por su sabor sino también por las propiedades nutritivas que posee, lo hace accesible a todo tipo de público (Barrientos, 2015). Los principales mercados para el grano de cacao ecuatoriano son: Estados Unidos, Holanda y Malasia con 93.3, 34.1 y 19.1 mil toneladas respectivamente, lo siguen países como México, Canadá, Alemania y otros, todos con menos del 12\% de participación (ANECACAO, 2015).

El cacao ha sido un cultivo tradicional desde la época de la colonia y ha sido fuente de trabajo de miles de familias que tienen esta actividad como su único sostén de ingresos económicos. La contribución en los años 2002-2011 al Producto Interno Bruto PIB total ha sido del $0.57 \%$ y al PIB Agropecuario del 6.4\%. Además, es un importante generador de empleo, se estima que aproximadamente 600,000 personas se involucran directamente en la actividad. La cadena de cacao participa con el 4\% de la Población Económicamente Activa (PEA) Nacional y el $12.5 \%$ de la PEA Agrícola, (Revista El Agro, 2017).

Los pequeños productores de cacao tipo Nacional se encuentran en una difícil situación socioeconómica debido a la baja productividad que presentan sus plantaciones originada por factores como grave impacto de las enfermedades y el bajo desempeño de muchas plantaciones por razones genéticas y de manejo (Phillips et al., 2012). Un mal tratamiento en post cosecha como mezcla, almacenamiento con otros productos pueden generar contaminación cruzada. Además, la falta de financiamiento para los pequeños productores es otro escenario que aqueja a este sector (Vasallo, 2015). Otra problemática identificada en la actividad cacaotera se da durante el proceso de comercialización, el autor antes mencionado señala que esta presenta una larga cadena de intermediación y de baja calidad. De esta forma el precio se ve mermado afectando directamente al pequeño productor.

Las formas de comercialización del cacao en Ecuador son para el mercado interno y externo. En cuanto a la comercialización externa, el país exporta en granos en sus dos variedades Sabor Arriba y Colección Castro Naranjal CCN51; los semielaborados como licor, manteca, torta, polvo y los elaborados como barras, tabletas, bombones, coberturas, polvo, relleno, baños y un sin fin de manufacturas más obtenidos a partir de mezclas con otros productos o frutos secos (ANECACAO, 2018). El mismo autor señala que en el año 2017 del cacao producido en Ecuador, el 87\% fueron exportaciones en grano, $12 \%$ semielaborados y un $0.8 \%$ correspondió a los productos terminados.

La comercialización interna se da cuando los intermediarios recorren las fincas para comprar directamente el producto en el estado en que se encuentre, generalmente secado al sol; después, el intermediario completa el proceso de secado mezclando varios tipos de cacao, ocasionando de esta forma pérdida de calidad. Otra manera de comercializar el cacao, es efectuando la venta a los acopiadores rurales de la zona, que reúnen lotes más amplios y a su vez lo venden a los mayoristas para que eventualmente sea entregado a los exportadores.

Un tercer circuito es cuando el agricultor va a los centros poblados más cercanos a vender su producto a intermediarios, quienes acopian el cacao en lotes más grandes para entregarlo al exportador. Una cuarta forma, es cuando el agricultor, generalmente el que tiene una propiedad más grande, vende su producto directamente al exportador (Morán y Villavicencio, 2008).

La práctica que se aplica en estas transacciones, es la calificación que realiza el comerciante al producto que consiste no solo en apreciación visual, sino que además toma un puñado de granos de cada saco, corta varias almendras para estimar el grado de fermentación, así como el porcentaje de humedad, que sirve para someter al productor a fuertes amonestaciones, que normalmente es descontado en el peso total del cacao. Además, la calidad del producto es seriamente afectada debido a prácticas inapropiadas en el procesamiento del grano, ya sea por falta de asesoramiento o incentivos, como la mezcla de variedades (CCN51 y Cacao Fino de Aroma) o la contaminación con elementos químicos o pesticidas que son restringidos en los mercados internacionales que prefieren optar por prácticas orgánicas (Zapata, 2017). Se debe incentivar la participación de los productores para implementar nuevas técnicas que contrarresten la poca productividad actual en las plantaciones y elevar la calidad del producto (De La Cruz et al., 2015).

Como alternativa para evitar a los intermediarios, algunos pequeños productores forman parte de asociaciones, los mismos que acumulan su producto y hacen exportación directa de cacao tipo orgánico certificado, obteniendo de esta forma un precio mayor. El sector agrícola es uno de los pocos sectores en que las asociaciones de productores son legales en la mayoría de los países, y con frecuencia se fomenta su creación, Además la inversión en asociaciones de productores puede reducir los costes de transacción, fortalecer la posición negociadora con los proveedores y estimular la producción en las explotaciones (Organización de las Naciones Unidas para 
la Alimentación y la Agricultura FAO, 2013). En Ecuador 90\% de los productores se encuentran registrados en asociaciones, pero no todos venden como parte de estas (Vasallo, 2015).

Con estos antecedentes el trabajo realizó un análisis de la cadena de comercialización del cacao Nacional producido por pequeños productores en la provincia de Los Ríos.

\section{Materiales y métodos}

T a investigación se realizó en provincia de Los Ríos, LEcuador, durante el mes de octubre del 2012, se entrevistó a pequeños productores de cacao tipo Nacional de la provincia de Los Ríos, usando para el efecto cuestionarios cuyas variables de estudio fueron los canales de distribución, precios y cantidades que se presentan en la comercialización del cacao.

Los agricultores participantes de la investigación fueron seleccionados mediante un muestreo por conveniencia, abarcando toda la provincia empleando criterios de dispersión geográficos, dividiendo la provincia en tres zonas: Norte, Central y Sur; así, la investigación se realizó en varios recintos pertenecientes a cada zona. Las entrevistas fueron aplicadas a 26 pequeños productores, distribuidos de la siguiente forma: nueve en la Zona Norte (Buena Fe y Valencia), seis en Zona Central (Mocache y Quinzaloma) y 11 en Zona Sur (Ventanas, Pueblo Viejo, Catarama y Baba). Para el análisis e interpretación de los datos registrados en las variables evaluadas, fue utilizada la estadística descriptiva determinando valores promedios, mínimos y máximos y en ciertos casos los porcentajes.

\section{Resultados y discusión}

Qn el cuadro 1 presenta el rango de edades de los - 1 productores entrevistados que va de 18 a 90 , con promedio de 62 años, lo que demuestra que los productores tienen gran experiencia en el cultivo de cacao, siendo en generalidad adultos mayores. En Ecuador son considerados adultos mayores, las personas con más de 65 años; bajo esta óptica, en el país hay 940,905 adultos, de los cuales, un 52.6\% son mujeres y el resto hombres (Instituto Nacional de Estadísticas y Censo INEC, 2010). La edad promedio de 62 años de los pequeños productores entrevistados (Cuadro 1), es elevada, si se compara con el promedio de 28 años que corresponde al promedio de edad de los habitantes de la provincia (INEC, 2010). Según Pocomucha (2016), este factor estaría afectando la producción de cacao, puesto que en el manejo del cultivo requiere esfuerzo físico, ya que la mayoría de los trabajos del campo se los realiza de forma manual. Esta situación, de elevado promedio de edad de los productores, se ha generalizado en varias regiones rurales de Latinoamérica, y no solo con productores de cacao, sino también con agricultores que se dedican a las distintas ramas agropecuarias.

Cuadro 1. Valores medios, mínimos y máximos de edad y sexo de los propietarios de las fincas de cacao de la provincia de Los Ríos, Ecuador (2012)

\begin{tabular}{lccc}
\hline & Edad & \multicolumn{2}{c}{ Sexo } \\
& (años) & Masculino & Femenino \\
\hline Porcentaje & - & 73.1 & 26.9 \\
Media & 62 & - & - \\
Mínimo & 18 & - & - \\
Máximo & 90 & - & - \\
\hline
\end{tabular}

Estos datos coinciden con la preocupación de Martínez (2007), quien manifiesta, que los campos del Ecuador están habitados en gran parte por adultos mayores, debidos que los jóvenes no quieren involucrarse en los trabajos del medio rural.

De los 26 propietarios entrevistados, el $73.1 \%$ fueron hombres y el $26.9 \%$ mujeres. De acuerdo con el INEC (2012), en la provincia de Los Ríos, el $83 \%$ de los productores son hombres el restante son mujeres, estableciéndose una relación de 8 a 2. Esto demuestra el aporte significativo de la mujer en la agricultura ecuatoriana. Las entrevistadas manifestaron que son ellas quienes realizan todas las labores que conlleva la producción de cacao e inclusive realizan la comercialización. De acuerdo a Ramírez (2006), la producción de cacao tiene todo un grupo de encadenamientos que inician a nivel de los productores individuales de grano de cacao y terminan en el mercado interno o externo; en este proceso están involucrados varios actores como las unidades de producción asociadas, los intermediarios, la industria casera, la industria de elaborados y los exportadores de cacao en grano.

En los resultados encontrados, el 89\% de los entrevistados realizan la comercialización por su cuenta, coincidiendo con Ramírez (2006), quien manifiesta que en el país el $90 \%$ de productores venden de forma individual y que son alrededor de 90,000 agricultores en su mayoría pequeños. En tanto que los intermediarios en Ecuador son alrededor de 1,000 con tamaños que dependen del volumen de compra y el lugar donde está ubicado el centro de acopio (MAGAP y FAO, 2010). 
La forma de operar de los intermediarios, muchas veces es concediendo créditos informales a los productores, mediante anticipos que van de 200 a 1,000 dólares y que son utilizados para cubrir gastos de mano de obra en las fincas. Estos préstamos se los entregan sin cobrar intereses y sin exigencias de garantía, se basa en la confianza y es a corto tiempo (UNOCACE, 2005). De esta forma el productor compromete de antemano su producción y si bien no paga intereses por el préstamo, tampoco puede discutir por los descuentos de porcentaje de humedad, impurezas y el precio a que está sometido.
La comercialización de los pequeños productores de cacao nacional en la provincia se realiza por medio de tres canales; el $76.9 \%$ vende al comerciante intermediario (de estos, el 55\% al intermediario que compra en la finca y el $45 \%$ al intermediario del poblado más cercano), el $11.5 \%$ vende directamente al exportador y el restante $11.5 \%$ entrega su cacao a asociaciones que se encargan de comercializar directamente (Figura 1). Este resultado coincide con lo expuesto por Jano y Mainville (2007), quienes indican que en el Ecuador el 70\% de los productores entregan su cosecha a los intermediarios, quienes hacen el trabajo de acumuladores de grano.

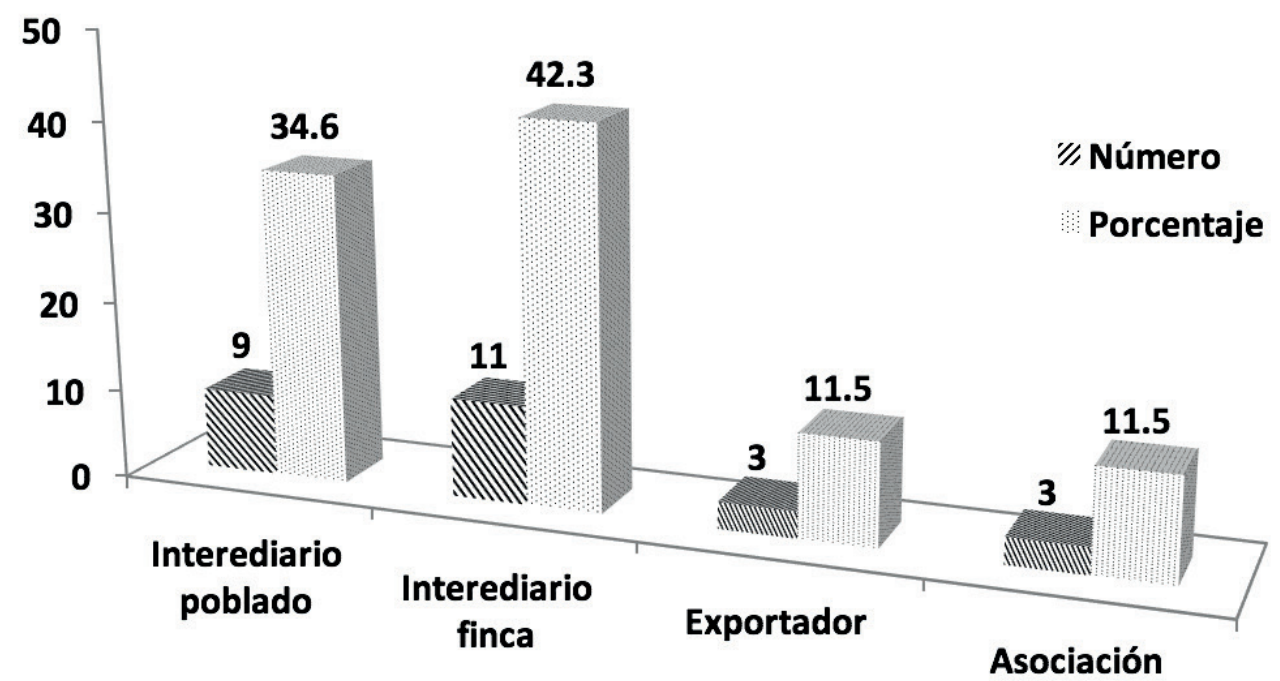

Figura 1. Número y porcentaje de pequeños productores de la provincia de Los Ríos que entregan el grano de cacao Nacional a los agentes comercializadores. 2012.

Al estar subordinada la comercialización de cacao de los pequeños productores a esta cadena de intermediarios, que compran en finca para entregar en los poblados, y estos a su vez a los mayoristas, quienes finalmente venden a los exportadores, el precio va disminuyendo ocasionando un perjuicio al productor. Acebedo et al. (2016) corrobora que la comercialización de cacao local involucra un gran número de acopiadores o intermediarios locales, a menudo ubicados en relaciones cautivas o trabajando a comisión para grandes comerciantes o subsidiarias de corporaciones multinacionales. El mismo autor señala que estos actores locales compiten agresivamente por los granos, llevando a la baja a los precios en la granja que reciben los productores locales.

Para UNOCACE (2005), los comerciantes retienen un valor, por concepto de utilidades y gastos generados, así, el autor expresa que: "El precio pagado al productor por el intermediario local depende del precio diario fijado por los comerciantes mayoristas y por los exportadores, dependiendo de este, cobran un margen de cinco dólares por saco de 45,45 kg para utilidades y cubrir gastos (mano de obra, local y alquiler del camión). También se toma en cuenta el riesgo asumido por el intermediario de una eventual caída del precio". De esta forma el productor que comercializa de forma individual, se encuentra sometido a las condiciones y precios que imponga el intermediario, perdiendo cualquier oportunidad de negociar y obtener un mejor precio.

El volumen de cacao comercializado por los pequeños productores es la totalidad de lo cosechado, en ocasiones semanal y otra mensual, donde la media de producción obtenida por los agricultores según la encuesta fue de $0.18 \mathrm{tha}^{-1}$ año ${ }^{-1}$. La producción obtenida por los agricultores contrasta grandemente con las estadísticas del MAGAP (2015), para la provincia de Los Ríos, quien reporta una producción de 0.36 t ha ${ }^{-1}$ año ${ }^{-1}$ para el año 2012. Esta diferencia significativa se debe a que las estadísticas del MAGAP contienen datos de rendimiento de cacao sin distinguir el Nacional del CCN51, que es una variedad más productiva, y en la provincia de Los Ríos, en los últimos años se ha propagado su siembra. Este hecho fue evidente durante el recorrido por los varios recintos de la provincia, donde se constató la gran cantidad de hectáreas sembradas con este material que sustituye el Nacional.

Otra forma de comercializar es por medio de las asociaciones, 
y en el Ecuador a escala micro, existen cerca de 30 asociaciones de agricultores activas que trabajan de manera articulada y participan en la producción, acopio y comercialización de cacao. Ellas dirigen el producto a intermediarios grandes, a la industria local o directamente a exportadores. En algunos casos concretos, estas asociaciones han incursionado en el mercado internacional (Ríos et al., 2017).

El $11.5 \%$ de los entrevistados que comercializan el cacao por medio de asociaciones respondieron que su producto lo entregan a organizaciones privadas, las que dan asesoría técnica en la producción y en varios casos entregan insumos para la plantación, resaltando que no tenían apoyo de organismos gubernamentales; sin embargo, son pocos los productores que están asociados y reciben ayuda del sector privado. Además, los productores destacaron como beneficio de estar asociados recibir entre 20 y 25 dólares más por saco de 45,45 $\mathrm{kg}$ de cacao. Esta afirmación está acorde con lo expuesto por Vasallo (2015) quien manifiesta que las asociaciones pagan un $30 \%$ por encima de los intermediarios. Siendo así, crear asociaciones se convierte en una alternativa para evadir a los intermediarios, cuyos beneficios obtenidos se podrían utilizar como un retorno para mejorar las labores en las fincas y por ende, mejorar la productividad.

En el cuadro 2 se observa las variaciones en el precio que han tenido las calidades de cacao. Para el año 2007 se muestra una marcada diferencia para las calidades del grano Nacional frente al CCN51. Al comparar el precio del grano conocido como triple $\mathrm{S}$ (ASSS) frente al cacao común CCN51, la variación es de 500 dólares más por tonelada, cantidad representativa para los escasos ingresos del productor.

Cuadro 2. Precio de exportación de cacao en grano, expresados en dólares por tonelada, desde el año 2007 hasta el 2011 (valor FOB )

\begin{tabular}{lccccccc}
\hline \multirow{2}{*}{ Calidad } & $\mathbf{2 0 0 7}$ & $\mathbf{2 0 0 8}$ & $\mathbf{2 0 0 9}$ & $\mathbf{2 0 1 0}$ & $\mathbf{2 0 1 1}$ & PROMEDIO & Variación \\
\cline { 2 - 6 } & \multicolumn{7}{c}{ Toneladas } \\
\hline ASE & $2,297.3$ & $2,481.5$ & $2,594.6$ & $2,900.4$ & $2,740.7$ & $2,602.9$ & 19,28 \\
ASN & & & $3,156.2$ & $3,143.2$ & $3,149.7$ & $-0,41$ \\
ASS & $2,454.9$ & $2,611.9$ & $2,775.1$ & $3,035.7$ & $2,934.2$ & $2,762.4$ & 19.55 \\
ASSPS & $2,550.3$ & $2,751.7$ & & & & $2,651.0$ & 7.88 \\
ASSS & $2,458.0$ & $2,606.0$ & $2,817.4$ & $3,085.9$ & $3,002.5$ & $2,794.0$ & 2.13 \\
CCN51 & $1,956.9$ & $2,303.5$ & $2,676.9$ & $2,887.9$ & $2,745.9$ & $2,514.2$ & 40.33 \\
\hline
\end{tabular}

Fuente: ANECACAO (2011).

Otro efecto encontrado, fue la calidad ASE en el transcurso de estos años prácticamente perdió su ventaja respecto al cacao CCN51. Cabe señalar que el agricultor no recibe pago por calidad, esta es exclusiva para el exportador, el comerciante normalmente paga por cacao Nacional y CCN51. La desaparición del pago por calidad, ha ocasionado que el agricultor reemplace una considerable cantidad de área destinada al cultivo de cacao Nacional por la variedad CCN51, que obtienen mayor producción y reciben casi el mismo precio. Menor productividad y mayor susceptibilidad a enfermedades que tienen los árboles productores de cacao fino respecto a otras variedades corrientes, ha determinado la decisión de escoger el tipo de material al momento de realizar nuevas siembras (Acebo et al., 2017).

El promedio de precio por tonelada del periodo analizado, muestra una diferencia de alrededor de 635 dólares del cacao ASN en comparación al CCN51 que obtiene el menor promedio; esto debido a que en los primeros años, el valor que se comercializaba tenía una diferencia marcada, cosa que no sucede en el último año, donde prácticamente el precio se ha equiparado con el cacao de calidad ASE. Sin embargo, al observar la variación que han tenido las calidades de cacao en el tiempo evaluado, el CCN51 presenta la más alta, alcanzando el $40.33 \%$ de incremento durante esos años

Todos los entrevistados que comercializan individualmente (89\%) manifestaron que no reciben pago por calidad de su producto y que muchos intermediarios pagaban el mismo precio al cacao Nacional y a la variedad CCN51. Uno de los entrevistados manifestó la decepción que sentía al momento de vender y encontrarse con precios que no sustentaban las inversiones realizadas en el cacao tipo Nacional. "No hay estímulos para el agricultor en los precios, hay una gran diferencia en producción entre las dos variedades, debería premiarse al agricultor por la calidad" (Entrevistado No 19 ). Con este sentir del agricultor, Acebo et al. (2016), concuerdan y expresan que una de las debilidades de la industria del cacao son los pocos incentivos para el productor del tipo Nacional que no recibe pago diferenciado de precios respecto a la variedad corriente.

Al analizar la figura 2 se observa la diferencia en precio que existe entre los eslabones de la cadena de comercialización de cacao Nacional, a la que están sujetos los pequeños agricultores, apreciando un margen de 8 dólares por saco entre el intermediario de finca y el intermediario del pueblo, corroborando lo indicado por Jano y Mainville (2008) quienes manifiestan que los intermediarios pagan un precio en finca 
de 6 a 8 dólares menos por saco, que si fuera vendido a los mismos intermediarios en el centro poblado más cercano y mucho menor al pagado por los exportadores.

El precio por saco de cacao recibido al comercializar mediante asociaciones es de 20 a 25 dólares mayor respecto al recibido cuando vende a los intermediarios de finca y los del pueblo.
Ríos et al. (2017) manifiestan que los productores asociados se han especializado en ofertar cacao con certificaciones y minimizan riesgos en el precio, además al cumplir con normas o programas requeridos por mercados especiales y obtienen un precio diferenciado sobre los convencionales.

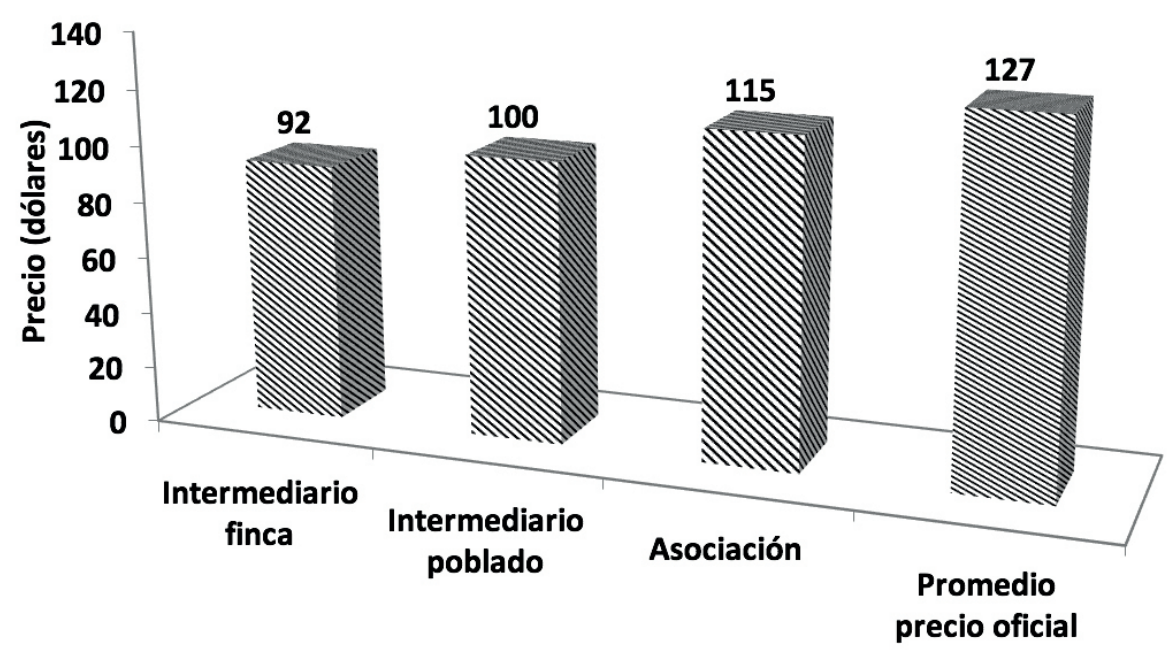

Figura 2. Precio por saco de cacao Nacional pagado por los diferentes agentes comercializadores. 2012.

\section{Conclusiones}

$\mathrm{L}$ a cadena de comercialización de cacao en la provincia

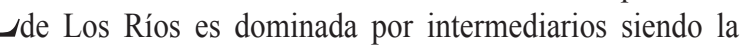
principal fuente de negociación para los pequeños productores. Una pequeña parte de los productores comercializa el cacao por medio de asociaciones y reciben un valor superior al pagado en el mercado, además de asesoría técnica. Como opción para mejorar los ingresos de los agricultores, se puede orientar en la formación de asociaciones que disminuye la problemática de comercialización evitando al intermediario. El precio del cacao es inestable, depende de varios factores entre ellos la demanda y a cuan larga sea la cadena para su comercialización. En los últimos años la diferencia en precio ha sido creciente debido a la gran demanda del mercado a nivel mundial, especialmente para el cacao ecuatoriano por ser en su mayoría fino y de aroma.

Es necesario mejorar los canales de distribución del pequeño productor por medio de programas de extensión y transferencia de tecnologías, capacitaciones, rehabilitación de plantaciones e innovación de productos derivados del cacao, obtenido mayores ingresos para el productor e impulsando el desarrollo local, regional y nacional.

\section{Bibliografía}

Acebo, M., Rodríguez, A., y Quijano, J., 2016. Estudios
Industriales, Orientación estratégica para la toma de decisión. Industria de cacao. Escuela Superior Politécnica del Litoral ESPOL.

ANECACAO (Asociación Nacional de Exportadores de Cacao). 2011. Precios mínimos referenciales F.O.B. para exportación de cacao \& semielaborados correspondiente a la semana del 27 de octubre al 2 de noviembre del 2011. http://www.anecacao.com/images/stories/precios $\% 20$ minimos $\% 20$ referenciales $\% 20$ del $\% 2027 \% 20$ oct $\% 20$ al\%202\%20nov.pdf. [5 noviembre 2011].

ANECACAO (Asociación Nacional de Exportadores de Cacao). 2015. Exportación ecuatoriana de cacao 2015. http:/www.anecacao.com/index.php/es/estadisticas/ estadisticas-actuales.html

Asociación Nacional de Exportadores de Cacao ANECACAO. 2018. Cacao Nacional. Un producto emblemático para el Ecuador. http://www.anecacao.com/index.php/es/ quienes-somos/cacao-nacional.html.

Barrientos, P. F. 2015. La cadena de valor del cacao en Perú y su oportunidad en el mercado mundial. Semestre Económico. 18 (37) 129-155.

De La Cruz, L., Córdova, E., García, N., Villanueva., Bucio A., y Villanueva, J. (2015). Manejo agronómico y caracterización socioeconómica del cacao en Comalcalco, Tabasco. Foresta Veracruzana. 17 (1) 33-44.

PROECUADOR (Instituto de Promoción de Exportaciones e Inversiones). 2013. Análisis del sector cacao y elaborados. 
INEC (Instituto Nacional de Estadísticas y Censos). 2010. Resultados del III Censo Nacional de Población en Ecuador. http://www.ecuadorencifras.gob.ec/censo-depoblacion-y-vivienda/ [10 abril 2015].

INEC (Instituto Nacional de Estadísticas y Censos). 2012. Infoeconomía. http://www.inec.gob.ec/inec/revistas/ info10.pdf [2 febrero 2015].

Jano, P. and Mainville, D. 2007. The cacao marketing chaing in Ecuador: Analysis of chain constraints to the development of markets for high-quality cacao. Parma, Italia, s.n.

Martínez, L. 2007. ¿Puede la pobreza rural ser abordada a partir de lo local?. Revista de Ciencias Sociales, Issue (29) 51-61.

MAGAP (Ministerio de Agricultura, Ganadería, Acuacultura y Pesca). 2012. MAGAP impulsa proyecto de reactivación del Cacao Fino y de Aroma. http://www.agricultura.gob. ec/magap-impulsa-proyecto-de-reactivacion-del-cacaofino-y-de-aroma/ [13 julio 2015].

MAGAP (Ministerio de Agricultura, Ganadería, Acuacultura y Pesca). 2015. Cacao: superficie, producción y rendimiento a nivel provincial. http://sinagap.agricultura. gob.ec/cacao/file/117-serie-historica-2000-2013 marzo 2016].

MAGAP (Ministerio de Agricultura, Ganadería, Acuacultura y Pesca); Organización de las Naciones Unidas para Agricultura y Alimentación FAO. 2010. Proyecto: Calidad de los alimentos vinculada con el origen y las tradiciones en América Latina, "Diagnóstico de la cadena del valor del cacao en el Ecuador".

Morán, I. y Villavicencio, J. 2008. Factibilidad de la producción y comercialización de cacao.

FAO (Organización de las Naciones Unidas para la Alimentación y la Agricultura). 2013. Agroindustrias para el desarrollo. Roma.

Phillips, W., Mora, A., Arciniegas-Leal, Allan., Mata-Quirós, J. y Motamayor-Arias. 2012. Catálogo de clones de cacao seleccionados por el Catie para siembras comerciales. Turrialba Costa Rica.

Pocomucha, V., Alegre, J., Abregú, L., 2016. Análisis socio económico y carbono almacenado en sistemas (Theobroma cacao L.) en Huánuco. Ecología Aplicada. 15. (2). 107-114.

Ramírez, P. 2006. Estructura y dinámica de la cadena de cacao en Ecuador. Sistematización y procesos en marcha. Quito: Cooperación Alemana de Desarrollo. Quito-Ecuador: GTZ.

Revista El Agro.2017. El cacao en la economía del Ecuador. http://www.revistaelagro.com/el-cacao-en-la-economiadel-ecuador/.

Ríos, F., Ruiz, A., Lecaro, J., Rehpani, C. (2017). Estrategias país para la oferta de cacaos especiales -Políticas e iniciativas privadas exitosas en el Perú, Ecuador, Colombia y República Dominicana. Fundación Swisscontact Colombia. Bogotá D. C. 140 p.

Rodríguez, D., Fusco, M. 2017. Gestión de riesgos agropecuarios en el sector del cacao en Ecuador. Revista de Investigación en Modelos Financieros - Año 6 (I) 57 $74 \mathrm{p}$.

UNOCACE (Unión de las Organizaciones Campesinas Cacaoteras del Ecuador). 2005. Estudio de la experiencia de la UNOCACE en la Cadena de Cacao en el Ecuador. Alianzas de aprendizaje.

Vasallo, M. 2015. Diferenciación y agregado de valor en la cadena ecuatoriana del cacao. 1 edición. IAEN. QuitoEcuador.

Zapata, A. 2017. Análisis de la producción de cacao fino de aroma en la provincia de Los Ríos. Facultad de Economía y Ciencias Empresariales. Universidad de Especialidades Espíritu Santo. 